\title{
On the Voros Coefficient for the Whittaker Equation with a Large Parameter - Some Progress around Sato's Conjecture in Exact WKB Analysis
}

\author{
by \\ Tatsuya KoIKe and Yoshitsugu TAKEI
}

\begin{abstract}
Generalizing Sato's conjecture for the Weber equation in exact WKB analysis, we explicitly determine the Voros coefficient of the Whittaker equation with a large parameter. By using our results we also compute alien derivatives of WKB solutions of the Whittaker equation at the so-called fixed singular points of their Borel transform.
\end{abstract}

2010 Mathematics Subject Classification: 34M30, 34M37, 34M55, 34M60.

Keywords: exact WKB analysis, Whittaker equation, Voros coefficient, alien derivative, Stokes automorphism.

\section{$\S 1$. Introduction}

In this article we study a Schrödinger equation

$$
\left(-\frac{d^{2}}{d x^{2}}+\eta^{2} Q(x, \eta)\right) \psi=0 \quad(\eta>0 \text { a large parameter })
$$

with the potential $Q(x, \eta)$ of the form

$$
Q(x, \eta)=\frac{1}{4}-\frac{\alpha}{x}+\eta^{-2} \frac{\beta}{x^{2}} \quad(\alpha, \beta \text { complex constants }),
$$

that is, the Whittaker equation with a large parameter $\eta$, from the viewpoint of exact WKB analysis.

This is a contribution to the special issue "The golden jubilee of algebraic analysis".

Communicated by M. Kashiwara. Received January 29, 2010. Revised April 2, 2010.

T. Koike: Department of Mathematics, Graduate School of Science, Kobe University,

1-1, Rokkodai, Nada-ku, Kobe 657-8501, Japan;

e-mail: koike@math.kobe-u.ac.jp

Y. Takei: RIMS, Kyoto University, Kyoto 606-8502, Japan;

e-mail: takei@kurims.kyoto-u.ac.jp

(C) 2011 Research Institute for Mathematical Sciences, Kyoto University. All rights reserved. 
In AKT2 Aoki, Kawai and the second author of the present article studied analytic properties of the Borel transforms of WKB solutions of an MTP equation, that is, a Schrödinger equation with a merging pair of simple turning points. Making use of the reduction of an MTP equation to a canonical one, we determined the location of "fixed singular points" (i.e., singular points whose relative locations with respect to the reference singular point are unchanged; cf. [DDP], [DP]) of Borel transformed WKB solutions and succeeded in explicitly computing the alien derivative of WKB solutions at fixed singular points for an MTP equation. In the case of an MTP equation the canonical equation is given by the Weber equation and what we call Sato's conjecture, that is, the explicit form of the Voros coefficient of the Weber equation in terms of the Bernoulli numbers (cf. [KT1]; see also SS] and [T] for its proof), played an important role in studying analytic properties of Borel transformed WKB solutions. (See, e.g., 2.13) in Section 2 for the definition of the Voros coefficient.)

On the other hand, as is emphasized in [Ko1] and [Ko2], the behavior of a WKB solution of a Schrödinger equation 1.1 near a simple pole is similar to that near a simple turning point. Inspired by these results, in a joint work [KKKoT1] with Kamimoto and Kawai we extended the analysis developed in AKT2 to an MPPT equation, that is, a Schrödinger equation with a merging pair of a simple pole and a simple turning point, again by making use of the reduction of an MPPT equation to a canonical one. In the case of an MPPT equation the canonical equation is given by the Whittaker equation and this motivates our interest in the Whittaker equation, and the computer-assisted study of its Voros coefficient indicated that it should be possible to write it down again in terms of the Bernoulli numbers. The aim of this paper is to show that it is really the case and to analyze the analytic structure of Borel transformed WKB solutions of the Whittaker equation by using the explicit form of the Voros coefficient thus obtained.

The paper is organized as follows: In Section 2 we present our main result, i.e., the precise formulation of the counterpart of Sato's conjecture for the Whittaker equation. In Section 3 we prove the main result. The proof follows an idea used in [T]. Finally in Section 4, by using the main result we study the analytic structure of the Borel transform of WKB solutions of the Whittaker equation.

\section{§2. Main Theorem}

The equation we discuss in this article is the Whittaker equation with a large parameter $\eta$ :

$$
\left\{-\frac{d^{2}}{d x^{2}}+\eta^{2}\left(\frac{1}{4}-\frac{\alpha}{x}+\eta^{-2} \frac{\beta}{x^{2}}\right)\right\} \psi=0 .
$$


Here $\alpha(\neq 0)$ and $\beta$ are complex constants. Our main interest is in the analysis of a WKB solution, that is, a formal solution of (2.1) of the form

$$
\psi=\exp \left(\int^{x} S(x, \eta) d x\right)
$$

where

$$
S(x, \eta)=\eta S_{-1}(x)+S_{0}(x)+\eta^{-1} S_{1}(x)+\cdots
$$

is a formal solution of the Riccati equation

$$
S^{2}+\frac{d S}{d x}=\eta^{2}\left(\frac{1}{4}-\frac{\alpha}{x}+\eta^{-2} \frac{\beta}{x^{2}}\right)
$$

associated to (2.1). Here we briefly review the construction of WKB solutions (cf. [KT2]). By substituting (2.3) into (2.4), we obtain the following recursion relations:

$$
\begin{gathered}
S_{-1}^{2}=\frac{1}{4}-\frac{\alpha}{x}, \\
2 S_{-1} S_{0}+\frac{d S_{-1}}{d x}=0, \\
2 S_{-1} S_{1}+S_{0}^{2}+\frac{d S_{0}}{d x}=\frac{\beta}{x^{2}}, \\
2 S_{-1} S_{n}+\sum_{j=0}^{n-1} S_{j} S_{n-j-1}+\frac{d S_{n-1}}{d x}=0 \quad(n \geq 2) .
\end{gathered}
$$

It follows from 2.5 that the leading term $S_{-1}(x)$ of 2.3 should be

$$
S_{-1}(x)= \pm \sqrt{\frac{1}{4}-\frac{\alpha}{x}}
$$

Once we fix the sign in 2.9 , i.e., the branch of $\sqrt{1 / 4-\alpha / x}$, the higher order terms $\left\{S_{n}\right\}_{n \geq 0}$ are determined uniquely and recursively by $2.6-2.8$. In particular, the sign in 2.9 is inherited only by the odd degree terms. Therefore there exist two formal solutions of 2.4 of the form

$$
S^{( \pm)}(x, \eta)= \pm S_{\text {odd }}(x, \eta)+S_{\text {even }}(x, \eta),
$$

where

$$
S_{\text {odd }}(x, \eta)=\sum_{j=0}^{\infty} \eta^{-2 j+1} S_{2 j-1}(x), \quad S_{\text {even }}(x, \eta)=\sum_{j=0}^{\infty} \eta^{-2 j} S_{2 j}(x) .
$$

Since

$$
S_{\text {even }}(x, \eta)=-\frac{1}{2} \frac{d}{d x} \log S_{\text {odd }}(x, \eta)
$$


(see Remark 2.2 in [KT2]), we thus obtain two WKB solutions of 2.1],

$$
\psi_{ \pm}(x, \eta)=\frac{1}{\sqrt{S_{\text {odd }}(x, \eta)}} \exp \left( \pm \int_{x_{0}}^{x} S_{\text {odd }}(x, \eta) d x\right) .
$$

In exact WKB analysis (cf. $[\mathrm{V}], \overline{\mathrm{DP}}$, $[\mathrm{KT} 2]$ ), we give an analytic meaning to these WKB solutions 2.2 or 2.12 through the Borel resummation method. As was explained in Introduction, the following theorem plays a crucial role in studying the analytic structure of Borel transformed WKB solutions.

Theorem 2.1. Let $V$ be the Voros coefficient of the Whittaker equation (2.1), that is,

$$
V:=\int_{4 \alpha}^{\infty}\left(S_{\text {odd }}(x, \eta)-\eta S_{-1}(x)\right) d x=\sum_{n=1}^{\infty} \eta^{1-2 n} \int_{4 \alpha}^{\infty} S_{2 n-1}(x) d x .
$$

Then the following relation holds as a formal power series in $\eta^{-1}$ :

$$
V=\sum_{n=1}^{\infty}(\alpha \eta)^{1-2 n} \frac{B_{2 n}(-\gamma)}{2 n \cdot(2 n-1)} .
$$

Here $\gamma$ is a constant satisfying $\gamma(\gamma+1)=\beta$ and $B_{n}(z)$ denotes the Bernoulli polynomial of degree $n$ defined by

$$
B_{n}(z)=\sum_{k=0}^{n} \frac{n !}{k !(n-k) !} B_{k} z^{n-k},
$$

where $\left\{B_{k}\right\}_{k \geq 0}$ are the Bernoulli numbers defined by the generating function

$$
\frac{t}{e^{t}-1}=\sum_{n=0}^{\infty} B_{n} \frac{t^{n}}{n !}
$$

Remark. (i) The lower endpoint $x=4 \alpha$ of the integration interval on the righthand side of 2.13) is a simple turning point of 2.1). Since $S_{n}(x)$ behaves like $O\left((x-4 \alpha)^{-1-3 n / 2}\right)$ near $x=4 \alpha, S_{2 n-1}(x)(n \geq 0)$ is not integrable near $4 \alpha$. We consider the integral in 2.13 as a contour integral along a path $\gamma$ shown in Figure 1, i.e.,

$$
V=\frac{1}{2} \int_{\gamma}\left(S_{\text {odd }}(x, \eta)-\eta S_{-1}(x)\right) d x .
$$

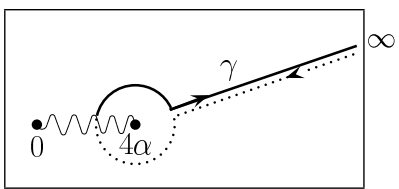

Figure 1 
Note also that, since we can show

$$
S_{n}(x)=O\left(|x|^{-1-n / 2}\right) \quad(|x| \rightarrow \infty)
$$

by induction on $n, S_{n}(x)$ with $n \geq 1$ is integrable near infinity.

(ii) The first few Bernoulli numbers are

$$
\begin{aligned}
& B_{0}=1, \quad B_{1}=-\frac{1}{2}, \quad B_{2 k+1}=0 \quad(k=1,2, \ldots), \\
& B_{2}=\frac{1}{6}, \quad B_{4}=-\frac{1}{30}, \quad B_{6}=\frac{1}{42}, \quad B_{8}=-\frac{1}{30}, \quad B_{10}=\frac{5}{66} .
\end{aligned}
$$

(iii) The generating function of the Bernoulli polynomials is given by

$$
\frac{t e^{t z}}{e^{t}-1}=\sum_{n=0}^{\infty} B_{n}(z) \frac{t^{n}}{n !} .
$$

Formula 2.18 is obtained by using the definition 2.15 of the Bernoulli polynomials and the generating function 2.16) of the Bernoulli numbers.

(iv) It is also known that the Bernoulli polynomial satisfies $B_{n}(1-z)=$ $(-1)^{n} B_{n}(z)$. In view of this relation we can readily find that the $2 n$-th Bernoulli polynomial $B_{2 n}(z)$ is a function of $z(z-1)$. Therefore the right-hand side of (2.14) is a function of $\beta$, not depending on the choice of a solution $\gamma$ of $\gamma(\gamma+1)=\beta$.

Theorem 2.1 is the counterpart of Sato's conjecture for the Whittaker equation. It is proved in Section 3 and by using Theorem 2.1 we study some analytic properties of the Borel transforms of WKB solutions in Section 4. Note that our results play an essential role in analyzing the fixed singular points of an MPPT equation in KKKoT1] (see also KKKoT2 for a review).

\section{$\S 3$. Proof of Main Theorem}

We prove Theorem 2.1 using the idea employed in [T]. Firstly, we derive the difference equation that the Voros coefficient satisfies. In this derivation the raising and lowering operators (with respect to the parameter $\alpha$ ) play a crucial role. Then, as the difference equation determines a solution uniquely, we compute its solution to verify that it coincides with the right-hand side of 2.14. In this paper we use the Borel transformation technique to compute a unique solution of the difference equation.

\section{§3.1. Derivation of the difference equation}

In this section the parameter $\alpha$ plays an important role. To show the dependence on $\alpha$ more manifestly, we use the notation $S^{( \pm)}(x, \alpha, \eta)$ for solutions 2.10 of the 
Riccati equation, $\psi_{ \pm}(x, \alpha, \eta)$ for WKB solutions, etc. in what follows. We also set

$$
I(\alpha, \eta):=\int_{\gamma}\left(S_{\text {odd }}(x, \alpha, \eta)-\eta S_{-1}(x, \alpha)\right) d x=2 V .
$$

In this subsection we prove

Proposition 3.1. I $(\alpha, \eta)$ formally satisfies

$$
\begin{aligned}
& I\left(\alpha+\eta^{-1}, \eta\right)-I(\alpha, \eta) \\
& =2+\log \frac{\alpha^{2}+\eta^{-1} \alpha-\eta^{-2} \beta}{\alpha^{2}}-2(\alpha \eta+1) \log \left(1+\frac{1}{\alpha \eta}\right) \\
& =2+\log \left[\left(1-\frac{\gamma}{\alpha \eta}\right)\left(1+\frac{\gamma+1}{\alpha \eta}\right)\right]-2(\alpha \eta+1) \log \left(1+\frac{1}{\alpha \eta}\right) .
\end{aligned}
$$

In Proposition 3.1 the word "formally" means the equality of formal power series with respect to $\eta^{-1}$. In fact, the right-hand side of 3.2 has a formal power series expansion

$$
-\frac{1+6 \beta}{\alpha^{2}} \eta^{-2}+\frac{1+6 \beta}{\alpha^{3}} \eta^{-3}-\frac{10 \beta^{2}+20 \beta+3}{20 \alpha^{4}} \eta^{-4}+\frac{15 \beta^{2}+15 \beta+2}{15 \alpha^{5}} \eta^{-5}+\cdots
$$

by the (formal) use of the Taylor expansion.

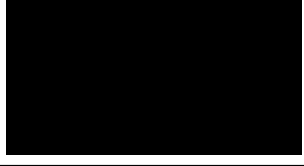

Figure 2

To prove Proposition 3.1. we first note that $S_{2 n}(x, \alpha)$ for $n=1,2, \ldots$ are single-valued around $x=4 \alpha$ and their residues are zero (cf. (2.11). Therefore

$$
I(\alpha, \eta)=\int_{\gamma}\left(S^{(+)}(x, \alpha, \eta)-\eta S_{-1}(x, \alpha)-S_{0}(x, \alpha)\right) d x .
$$

In studying the right-hand side of (3.4), we also consider the following cut-off integrals:

$$
\begin{aligned}
I^{x}(\alpha, \eta) & =\int_{\gamma_{x}} S^{(+)}(x, \alpha, \eta) d x, \\
I_{n}^{x}(\alpha, \eta) & =\int_{\gamma_{x}} S_{n}(x, \alpha) d x \quad(n=-1,0),
\end{aligned}
$$


where $\gamma_{x}$ is the contour shown in Figure 2. From (3.4) and the definition of $I^{x}$ and $I_{n}^{x}$, we have

$$
I(\alpha, \eta)=\lim _{x \rightarrow \infty}\left[I^{x}(\alpha, \eta)-\eta I_{-1}^{x}(\alpha)-\eta I_{0}^{x}(\alpha)\right] .
$$

We then determine the asymptotic behavior of $I^{x}\left(\alpha+\eta^{-1}, \eta\right)-I^{x}(\alpha, \eta)$.

Lemma 3.1. As $x$ tends to infinity, we have

$$
I^{x}\left(\alpha+\eta^{-1}, \eta\right)-I^{x}(\alpha, \eta)=\log \frac{\alpha^{2}+\eta^{-1} \alpha-\eta^{-2} \beta}{x^{2}}+O\left(|x|^{-1}\right) .
$$

To prove Lemma 3.1, we use the raising and lowering operators of the Whittaker equation with respect to $\alpha$ :

$$
\mathcal{A}(\alpha)=\eta^{-1} \frac{d}{d x}+\frac{1}{2} x-\alpha, \quad \mathcal{A}^{\dagger}(\alpha)=\eta^{-1} \frac{d}{d x}-\frac{1}{2} x+\alpha .
$$

We also set

$$
L(\alpha)=\frac{d^{2}}{d x^{2}}-\eta^{2}\left(\frac{1}{4}-\frac{\alpha}{x}+\eta^{-2} \frac{\beta}{x^{2}}\right) .
$$

Lemma 3.2. If $\psi$ is a solution of the Whittaker equation (2.1) (i.e. $L(\alpha) \psi=0$ ), then $L\left(\alpha-\eta^{-1}\right) \mathcal{A}(\alpha) \psi=0$ and $L\left(\alpha+\eta^{-1}\right) \mathcal{A}^{\dagger}(\alpha) \psi=0$.

Proof. By direct computations, we have

$$
\eta^{-2} x^{2} L(\alpha)=\mathcal{A}\left(\alpha+\eta^{-1}\right) \mathcal{A}^{\dagger}(\alpha)+c(\alpha)=\mathcal{A}^{\dagger}\left(\alpha-\eta^{-1}\right) \mathcal{A}(\alpha)+c\left(\alpha-\eta^{-1}\right)
$$

with

$$
c(\alpha)=\alpha^{2}+\eta^{-1} \alpha-\eta^{-2} \beta .
$$

Therefore

$$
\begin{aligned}
\eta^{-2} x^{2} L\left(\alpha+\eta^{-1}\right) \mathcal{A}^{\dagger}(\alpha) \psi & =\left[\mathcal{A}^{\dagger}(\alpha) \mathcal{A}\left(\alpha+\eta^{-1}\right)+c(\alpha)\right] \mathcal{A}^{\dagger}(\alpha) \psi \\
& =\left[\mathcal{A}^{\dagger}(\alpha) \mathcal{A}\left(\alpha+\eta^{-1}\right) \mathcal{A}^{\dagger}(\alpha)+c(\alpha) \mathcal{A}^{\dagger}(\alpha)\right] \psi \\
& =\left[\mathcal{A}^{\dagger}(\alpha)\left\{\eta^{-2} x^{2} L(\alpha)-c(\alpha)\right\}+c(\alpha) \mathcal{A}^{\dagger}(\alpha)\right] \psi=0 .
\end{aligned}
$$

By the same reasoning we can also prove $\eta^{-2} x^{2} L\left(\alpha-\eta^{-1}\right) \mathcal{A}(\alpha) \psi=0$.

Proof of Lemma 3.1. Lemma 3.2 implies that there exists a constant $C(\eta)$ for which

$$
\left(\eta^{-1} x \frac{d}{d x}-\frac{1}{2} x+\alpha\right) \psi_{+}(x, \alpha, \eta)=C(\eta) \psi_{+}\left(x, \alpha+\eta^{-1}, \eta\right) .
$$

Since the left-hand side of (3.7) is

$$
\left(\eta^{-1} x S^{(+)}-\frac{1}{2} x+\alpha\right) \psi_{+}(x, \alpha, \eta),
$$


the logarithmic derivatives of both sides of (3.7) give

$$
S^{(+)}\left(x, \alpha+\eta^{-1}, \eta\right)=S^{(+)}(x, \alpha, \eta)+\frac{d}{d x} \log \left[\eta^{-1} x S^{(+)}(x, \alpha, \eta)-\frac{1}{2} x+\alpha\right] .
$$

Therefore we obtain

$$
\begin{aligned}
& \int_{\gamma_{x}} S^{(+)}\left(x, \alpha+\eta^{-1}, \eta\right) d x-\int_{\gamma_{x}} S^{(+)}(x, \alpha, \eta) d x \\
& =\log \left[\eta^{-1} x S^{(+)}(x, \alpha, \eta)-\frac{1}{2} x+\alpha\right]-\log \left[\eta^{-1} x S^{(-)}(x, \alpha, \eta)-\frac{1}{2} x+\alpha\right] .
\end{aligned}
$$

To determine the asymptotic behavior of the right-hand side of $(3.8)$, we use the following explicit formulas as $x \rightarrow \infty$ which are easily obtained from the recursion relations (2.5)-2.8):

$$
\begin{aligned}
S_{-1}(x, \alpha)= & \sqrt{\frac{1}{4}-\frac{\alpha}{x}}=\frac{1}{2}-\frac{\alpha}{x}-\frac{\alpha^{2}}{x^{2}}-\frac{2 \alpha^{3}}{x^{3}}+O\left(|x|^{-4}\right) \\
S_{0}(x, \alpha)= & \frac{1}{4}\left(\frac{1}{x}-\frac{1}{x-4 \alpha}\right)=-\frac{\alpha}{x^{2}}+O\left(|x|^{-3}\right) \\
S_{1}(x, \alpha)= & -\frac{5}{16} x^{1 / 2}(x-4 \alpha)^{-5 / 2}+\frac{1}{8} x^{-1 / 2}(x-4 \alpha)^{-3 / 2} \\
& +\left(\beta+\frac{3}{16}\right) x^{-3 / 2}(x-4 \alpha)^{-1 / 2} \\
= & \frac{\beta}{x^{2}}+O\left(|x|^{-3}\right) .
\end{aligned}
$$

These asymptotic formulas together with 2.17) show

$$
\begin{aligned}
& S^{(+)}(x, \alpha, \eta)=\eta\left(\frac{1}{2}-\frac{\alpha}{x}-\frac{\alpha^{2}}{x^{2}}\right)+\frac{-\alpha+\eta^{-1} \beta}{x^{2}}+O\left(|x|^{-5 / 2}\right), \\
& S^{(-)}(x, \alpha, \eta)=\eta\left(-\frac{1}{2}+\frac{\alpha}{x}+\frac{\alpha^{2}}{x^{2}}\right)+\frac{-\alpha-\eta^{-1} \beta}{x^{2}}+O\left(|x|^{-5 / 2}\right) .
\end{aligned}
$$

The formula 3.6 readily follows from $3.8,3.9$ and 3.10 . This completes the proof of Lemma 3.1 .

Next we determine the asymptotic behavior of $I_{-1}^{x}\left(\alpha+\eta^{-1}\right)-I_{-1}^{x}(\alpha)$ and $I_{0}^{x}\left(\alpha+\eta^{-1}\right)-I_{0}^{x}(\alpha)$.

Lemma 3.3. (i) As $x$ tends to infinity, we have

$$
I_{-1}^{x}\left(\alpha+\eta^{-1}\right)-I_{-1}^{x}(\alpha)=\eta^{-1}\left[-2+2(1+\alpha \eta) \log \left(1+\frac{1}{\alpha \eta}\right)+\log \frac{\alpha^{2}}{x^{2}}\right]+O\left(|x|^{-1}\right) .
$$

(ii) $I_{0}^{x}\left(\alpha+\eta^{-1}\right)-I_{0}^{x}(\alpha)=0$. 
Proof. Since $S_{0}(x)$ is single-valued near $x=4 \alpha, I_{0}^{x}(\alpha)$ is $2 \pi i$ times the residue of $S_{0}(x)$ at $x=4 \alpha$. Hence $I_{0}^{x}(\alpha)=-i \pi / 2$ and thus we obtain (ii). To prove (i), we compute the integral explicitly:

$$
\begin{aligned}
I_{-1}^{x}(\alpha)= & \int_{\gamma_{x}} S_{-1} d x \\
= & 2 x \sqrt{\frac{1}{4}-\frac{\alpha}{x}}-\alpha \log \left[x-2 \alpha+2 x \sqrt{\frac{1}{4}-\frac{\alpha}{x}}\right] \\
& +\alpha \log \left[x-2 \alpha-2 x \sqrt{\frac{1}{4}-\frac{\alpha}{x}}\right] .
\end{aligned}
$$

As $x$ tends to infinity, we have

$$
\sqrt{\frac{1}{4}-\frac{\alpha}{x}}=\frac{1}{2}-\frac{\alpha}{x}+\frac{\alpha^{2}}{x^{2}}+O\left(|x|^{-3}\right)
$$

and hence

$$
\begin{aligned}
& x-2 \alpha+2 x \sqrt{\frac{1}{4}-\frac{\alpha}{x}}=2 x-4 \alpha-\frac{2 \alpha^{2}}{x}+O\left(|x|^{-2}\right), \\
& x-2 \alpha-2 x \sqrt{\frac{1}{4}-\frac{\alpha}{x}}=\frac{2 \alpha^{2}}{x}+O\left(|x|^{-2}\right) .
\end{aligned}
$$

Therefore we obtain

$$
I_{-1}^{x}(\alpha)=\int_{\gamma_{x}} S_{-1}(x, \alpha) d x=x-2 \alpha+\alpha \log \frac{\alpha^{2}}{x^{2}}+O\left(|x|^{-1}\right) .
$$

This asymptotic behavior of $I_{-1}^{x}(\alpha)$ verifies (i).

We now prove Proposition 3.1.

Proof of Proposition 3.1. Lemmas 3.1 and 3.2 give

$$
\begin{aligned}
& {\left[I^{x}\left(\alpha+\eta^{-1}, \eta\right)-\eta I_{-1}^{x}\left(\alpha+\eta^{-1}\right)-I_{0}^{x}\left(\alpha+\eta^{-1}\right)\right]-\left[I^{x}(\alpha, \eta)-\eta I_{-1}^{x}(\alpha)-I_{0}^{x}(\alpha)\right]} \\
& \quad=\left[I^{x}\left(\alpha+\eta^{-1}, \eta\right)-I^{x}(\alpha, \eta)\right]-\eta\left[I_{-1}^{x}\left(\alpha+\eta^{-1}\right)-I_{-1}^{x}(\alpha)\right] \\
& \quad=2-2(1+\alpha \eta) \log \left(1+\frac{1}{\alpha \eta}\right)+\log \frac{\alpha^{2}+\eta^{-1} \alpha-\eta^{-2} \beta}{\alpha^{2}}+O\left(|x|^{-1}\right) .
\end{aligned}
$$

This relation and 3.5 give 3.2 . Since $\beta=\gamma(\gamma+1)$, we can also verify

$$
\frac{\alpha^{2}+\eta^{-1} \alpha-\eta^{-2} \beta}{\alpha^{2}}=\left(1-\frac{\gamma}{\alpha \eta}\right)\left(1+\frac{\gamma+1}{\alpha \eta}\right) .
$$

This proves 3.3 . 


\section{§3.2. Determination of the Voros coefficient}

Let $V(\alpha, \eta)$ be the Voros coefficient defined by 2.13 . From Proposition 3.1 and (3.1), we have

$$
\begin{aligned}
& V\left(\alpha+\eta^{-1}, \eta\right)-V(\alpha, \eta) \\
& \quad=1+\frac{1}{2} \log \left[\left(1-\frac{\gamma}{\alpha \eta}\right)\left(1+\frac{\gamma+1}{\alpha \eta}\right)\right]-(\alpha \eta+1) \log \left(1+\frac{1}{\alpha \eta}\right) .
\end{aligned}
$$

In this subsection we verify that this difference equation determines $V$ uniquely. To this end we convert (3.11) to a difference equation with respect to $\eta$ by using the homogeneity of $V(\alpha, \eta)$.

Lemma 3.4. Let

$$
V_{2 n-1}(\alpha)=\int_{4 \alpha}^{\infty} S_{2 n-1}(x, \alpha) d x \quad(n=1,2, \ldots) .
$$

Then

$$
V_{2 n-1}(\alpha)=\alpha^{1-2 n} V_{2 n-1}(1) \quad \text { for } n=1,2, \ldots
$$

Proof. Using the recursion relations 2.5 -2.8 , we can verify

$$
S_{n}(\alpha x, \alpha)=\frac{1}{\alpha^{n+1}} S_{n}(x, 1) \quad(n=-1,0,1,2, \ldots)
$$

by induction on $n$. Therefore

$$
\int_{4 \alpha}^{\infty} S_{2 n-1}(x, \alpha) d x=\alpha \int_{4}^{\infty} S_{2 n-1}(\alpha t, \alpha) d t=\alpha^{1-2 n} \int_{4}^{\infty} S_{2 n-1}(t, 1) d t
$$

for $n=1,2, \ldots$.

Thanks to Lemma 3.4 .

$$
V(\alpha, \eta)=\sum_{n=1}^{\infty} V_{2 n-1}(\alpha) \eta^{1-2 n}=\sum_{n=1}^{\infty} V_{2 n-1}(1)(\alpha \eta)^{1-2 n},
$$

and

$$
\left(\alpha+\eta^{-1}\right) \eta=\alpha \eta+1=\alpha\left(\eta+\frac{1}{\alpha}\right)
$$

entails

$$
V\left(\alpha+\eta^{-1}, \eta\right)=V\left(\alpha, \eta+\frac{1}{\alpha}\right) .
$$

From (3.12) and (3.11) we obtain the following difference equation for $V$ with respect to $\eta$. 


\section{Proposition 3.2.}

$$
\begin{aligned}
& V\left(\alpha, \eta+\frac{1}{\alpha}\right)-V(\alpha, \eta) \\
& \quad=1+\frac{1}{2} \log \left[\left(1-\frac{\gamma}{\alpha \eta}\right)\left(1+\frac{\gamma+1}{\alpha \eta}\right)\right]-(\alpha \eta+1) \log \left(1+\frac{1}{\alpha \eta}\right)
\end{aligned}
$$

We solve the difference equation 3.13 by using the Borel transformation. We first note that 3.13 has a unique (formal) solution of the form

$$
V=\sum_{n=1}^{\infty} V_{n} \eta^{1-2 n}=V_{1} \eta^{-1}+V_{3} \eta^{-3}+V_{5} \eta^{-5}+\cdots
$$

Hence (3.13) determines $V$ uniquely. We now explicitly compute its Borel transform

$$
V_{B}=\sum_{n=1}^{\infty} \frac{V_{n}}{(2 n-2) !} y^{2 n-2}
$$

by considering the Borel transform of 3.13 . The Borel transform of the left-hand side of 3.13 is

$$
\left(e^{-y / \alpha}-1\right) V_{B}(\alpha, y)
$$

To compute the Borel transform of the right-hand side, we use

Lemma 3.5. Let $C$ be a non-zero complex constant. Then

(i) $\mathcal{B}\left[\log \left(1+C \eta^{-1}\right)\right](y)=\frac{1-e^{-C y}}{y}$.

(ii) $\mathcal{B}\left[\eta \log \left(1+C \eta^{-1}\right)-C\right](y)=\frac{-1+(1+C y) e^{-C y}}{y^{2}}$.

Here $\mathcal{B}$ denotes the Borel transformation with respect to $\eta$.

Lemma 3.5 can be proved by straightforward computations. By using Lemma 3.5 we can compute the Borel transform of the right-hand side of 3.13 in the following way:

$$
\begin{aligned}
\mathcal{B}[1-(\alpha \eta+1) & \left.\log \left(1+\frac{1}{\alpha \eta}\right)\right] \\
= & -\mathcal{B}\left[\alpha\left(\eta \log \left(1+\frac{1}{\alpha \eta}\right)-\frac{1}{\alpha}\right)\right]-\mathcal{B}\left[\log \left(1+\frac{1}{\alpha \eta}\right)\right] \\
= & \frac{\alpha}{y^{2}}\left[1-\left(1+\frac{y}{\alpha}\right) e^{-y / \alpha}\right]-\frac{1-e^{-y / \alpha}}{y} \\
= & \alpha \frac{1-e^{-y / \alpha}}{y^{2}}-\frac{1}{y}
\end{aligned}
$$




$$
\begin{aligned}
\mathcal{B}\left[\frac{1}{2} \log \left(1-\frac{\gamma}{\alpha \eta}\right)(\right. & \left.\left.1+\frac{\gamma+1}{\alpha \eta}\right)\right] \\
& =\frac{1}{2} \mathcal{B}\left[\log \left(1-\frac{\gamma}{\alpha \eta}\right)\right]+\frac{1}{2} \mathcal{B}\left[\log \left(1+\frac{\gamma+1}{\alpha \eta}\right)\right] \\
& =\frac{1}{2} \cdot \frac{1-e^{\gamma y / \alpha}}{y}+\frac{1}{2} \cdot \frac{1-e^{-(\gamma+1) y / \alpha}}{y} \\
& =\frac{1}{y}-\frac{1}{2} \cdot \frac{e^{\gamma y / \alpha}+e^{-(\gamma+1) y / \alpha}}{y} .
\end{aligned}
$$

We conclude from (3.14, 3.15 and 3.16 ) that the difference equation 3.13 becomes

$$
\left(e^{-y / \alpha}-1\right) V_{B}(\alpha, y)=\alpha \frac{1-e^{-y / \alpha}}{y^{2}}-\frac{1}{2} \cdot \frac{e^{\gamma y / \alpha}+e^{-(\gamma+1) y / \alpha}}{y}
$$

after the Borel transformation. This equation can be easily solved and we obtain

Theorem 3.1. The Borel transform $V_{B}$ of the Voros coefficient $V$ is given by

$$
V_{B}(\alpha, y)=\frac{1}{2 y} \cdot \frac{e^{-\gamma y / \alpha}+e^{(\gamma+1) y / \alpha}}{e^{y / \alpha}-1}-\frac{\alpha}{y^{2}} .
$$

Thus, to prove Theorem 2.1. it suffices to show that the Borel transform of the right-hand side of (2.14) coincides with the right-hand side of (3.17). The Borel transform of the right-hand side of 2.14 is, by its definition,

$$
\sum_{n=1}^{\infty} \alpha^{1-2 n} \frac{B_{2 n}(-\gamma)}{(2 n) !} y^{2 n-2}=\frac{\alpha}{y^{2}} \sum_{n=1}^{\infty} \frac{B_{2 n}(-\gamma)}{(2 n) !}\left(\frac{y}{\alpha}\right)^{2 n} .
$$

We then use 2.18 to obtain

$$
\sum_{n=0}^{\infty} \frac{B_{2 n}(z)}{(2 n) !} t^{2 n}=\frac{1}{2}\left[\frac{t e^{t z}}{e^{t}-1}+\frac{-t e^{-t z}}{e^{-t}-1}\right]=\frac{t}{2} \cdot \frac{e^{t z}+e^{-t(z-1)}}{e^{t}-1} .
$$

From (3.18) and (3.19) we can confirm that the Borel transform of the right-hand side of (2.14) is exactly the same as the right-hand side of (3.17). This proves Theorem 2.1.

Remark. Since

$$
\begin{aligned}
e^{-\gamma y / \alpha}+ & e^{(\gamma+1) y / \alpha} \\
& =\frac{1}{2}\left[\left(e^{y / \alpha}+1\right)\left(e^{\gamma y / \alpha}+e^{-\gamma y / \alpha}\right)+\left(e^{y / \alpha}-1\right)\left(e^{\gamma y / \alpha}-e^{-\gamma y / \alpha}\right)\right] \\
& =\left(e^{y / \alpha}+1\right) \cosh \frac{\gamma y}{\alpha}+\left(e^{y / \alpha}-1\right) \sinh \frac{\gamma y}{\alpha}
\end{aligned}
$$


we have the following expression for $V_{B}$ :

$$
V_{B}=\frac{1}{2}\left[\frac{1}{y} \frac{e^{y / \alpha}+1}{e^{-y / \alpha}-1} \cosh \frac{\gamma y}{\alpha}+\frac{1}{y} \sinh \frac{\gamma y}{\alpha}-\frac{2 \alpha}{y^{2}}\right] .
$$

This expression was used in KKKoT1 and [KKKoT2].

The following lemma will be used in the subsequent section.

Proposition 3.3. $V_{B}$ has poles at $y=2 m \pi i \alpha(m \in \mathbb{Z} \backslash\{0\})$ and has no other singular points. The poles are all simple with residues

$$
\underset{y=2 m \pi i \alpha}{\operatorname{Res}} V_{B}=\frac{e^{2 m \pi i \gamma}+e^{-2 m \pi i \gamma}}{4 m \pi i} .
$$

\section{§4. Analytic structure of the Borel transform of WKB solutions for the Whittaker equation}

In this section we derive some important analytic properties of the Borel transforms of WKB solutions of the Whittaker equation. All of them are obtained as consequences of our main theorems (Theorems 2.1 and 3.1. For simplicity we restrict our considerations in this section to the case where $\arg \alpha=\pi / 2$ or $\arg \alpha$ is sufficiently close to $\pi / 2$. The configuration of Stokes curves, i.e., integral curves of the direction field $\Im\left(S_{-1}(x) d x\right)=0$ emanating from turning points, of the Whittaker equation 2.1 for $\arg \alpha=\pi / 2$ is shown in Figure 3. Note that the origin also plays the same role as a turning point (cf. [Ko1] and [Ko2]). We also show in Figure 4 and Figure 6 (resp., Figure 5 and Figure 7 ) the configuration of Stokes curves for $\arg \alpha=\pi / 2-\varepsilon$ (resp., $\arg \alpha=\pi / 2+\varepsilon$ ) for sufficiently small positive $\varepsilon$.

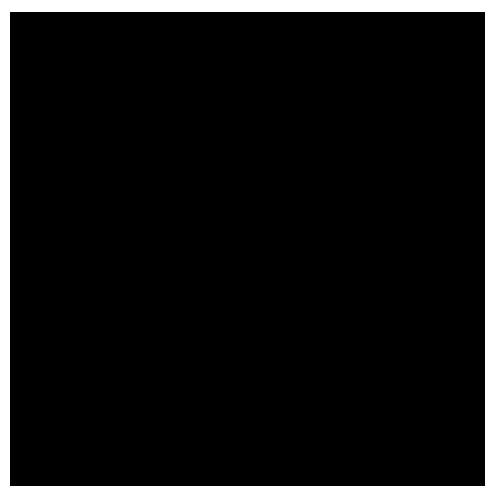

Figure 3. $\arg \alpha=\pi / 2$ 


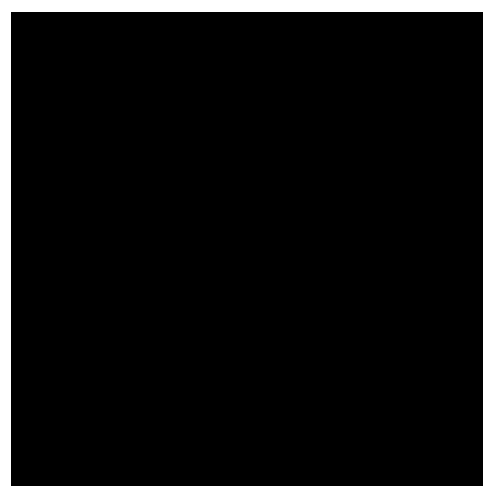

Figure 4. $\arg \alpha=\pi / 2-0.2$

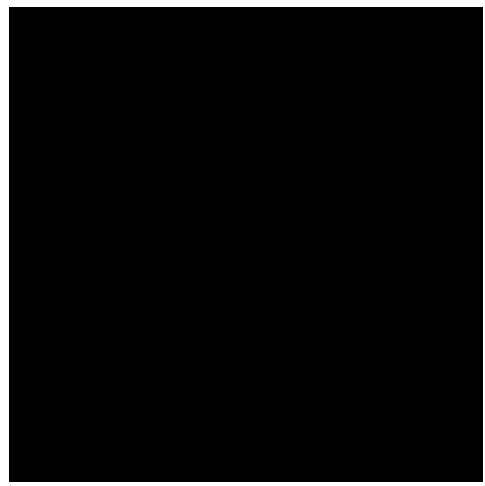

Figure $6 . \arg \alpha=\pi / 2-0.45$

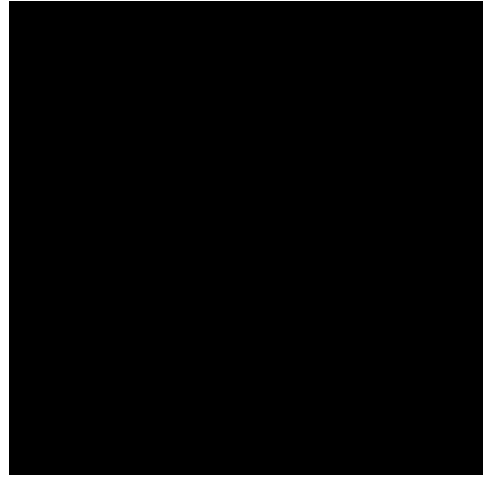

Figure 5. $\arg \alpha=\pi / 2+0.2$

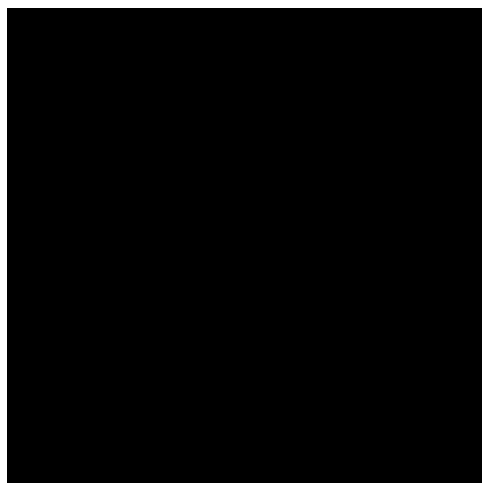

Figure 7. $\arg \alpha=\pi / 2+0.45$

As we can observe from these figures, degeneration occurs in the configuration of Stokes curves for $\arg \alpha=\pi / 2$ (Figure 3), i.e., there exists a Stokes curve connecting a turning point $4 \alpha$ and the origin in Figure 3. Although this degeneration is resolved in Figures 4-7, the topological configuration of Stokes curves is quite different between, for example, Figure 6 and Figure 7; in particular, the configuration of a Stokes curve emanating from $4 \alpha$ abruptly changes at $\arg \alpha=\pi / 2$. We will see in $\S 4.2$ that this abrupt change of the configuration of Stokes curves is related to Stokes phenomena for WKB solutions that occur when $\alpha$ varies.

\section{§4.1. Fixed singularities of the Borel transforms of WKB solutions}

Let us consider WKB solutions normalized at a simple turning point $4 \alpha$ :

$$
\psi_{ \pm}=\frac{1}{\sqrt{S_{\text {odd }}}} \exp \left[ \pm \int_{4 \alpha}^{x} S_{\text {odd }} d x\right]
$$


These WKB solutions $\psi_{+}$and $\psi_{-}$can be expanded as

$$
\psi_{ \pm}=e^{\eta y_{ \pm}(x)} \sum_{n=0}^{\infty} \psi_{ \pm, n}(x) \eta^{-n-1 / 2}
$$

with

$$
y_{ \pm}(x)= \pm \int_{4 \alpha}^{x} S_{-1}(x) d x= \pm \int_{4 \alpha}^{x} \sqrt{\frac{1}{4}-\frac{\alpha}{x}} d x
$$

and the Borel transform $\psi_{ \pm, B}$ of $\psi_{ \pm}$is given by

$$
\psi_{ \pm, B}(x, y)=\sum_{n=0}^{\infty} \frac{\psi_{ \pm, n}(x)}{\Gamma(n+1 / 2)}\left(y+y_{ \pm}(x)\right)^{n-1 / 2} .
$$

Theorem 4.1. Assume that the path of integration in 4.1 does not cross any Stokes curve for any $\alpha$ with $|\arg \alpha-\pi / 2|<\varepsilon(\varepsilon>0)$. Then the Borel transform of the WKB solution $\psi_{+, B}$ (resp., $\psi_{-, B}$ ) has singular points at $y=-y_{+}(x)+2 m \pi i \alpha$ (resp., $\left.y=-y_{-}(x)+2 m \pi i \alpha\right)$ with $m \in \mathbb{Z} \backslash\{0\}$. Furthermore, their alien derivatives there are given by

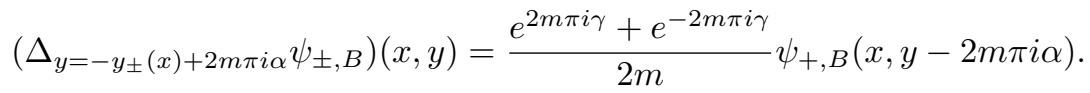

Note that the singular points described in Theorem 4.1 are fixed singular points (cf. $\mathrm{DDP},[\mathrm{DP}]$ ) of $\psi_{+}$(resp., $\psi_{-}$) since their relative locations with respect to the reference singular point $y=-y_{+}(x)$ (resp., $y=-y_{-}(x)$ ) are unchanged when $x$ varies.

In this subsection we prove Theorem 4.1. Since the same reasoning holds also for $\psi_{-, B}$, we only consider $\psi_{+, B}$. We refer the reader to Sa for the definition of the alien derivative and the relevant alien calculus employed in what follows. See also [AKT2, §3] where a similar discussion is given for the Weber equation.

To prove Theorem 4.1 we use the relation

$$
\psi_{+}=\exp \left[\int_{4 \alpha}^{\infty}\left(S_{\text {odd }}-\eta S_{-1}(x)\right) d x\right] \psi_{+}^{(\infty)}=e^{V} \psi_{+}^{(\infty)},
$$

where $\psi_{+}^{(\infty)}$ is a WKB solution normalized at infinity:

$$
\psi_{+}^{(\infty)}=\frac{1}{\sqrt{S_{\text {odd }}}} \exp \left[\eta \int_{4 \alpha}^{x} S_{-1}(x) d x\right] \exp \left[\int_{\infty}^{x}\left(S_{\text {odd }}(x, \eta)-\eta S_{-1}(x)\right) d x\right] .
$$

The formal relation 4.3 becomes

$$
\psi_{+, B}=\mathcal{B}\left[e^{V}\right] * \psi_{+, B}^{(\infty)}
$$


after the Borel transformation, where $*$ denotes the convolution product for the variable $y$. Since $V_{B}$ is singular at $y=2 m \pi i \alpha(m \in \mathbb{Z} \backslash\{0\})$ as we have seen in Section 3 , we then find that $y=2 m \pi i \alpha$ is also a singular point of $\psi_{+, B}$.

To compute the alien derivative of $\psi_{+}$there, we use

Lemma 4.1. The WKB solution (4.4) normalized at infinity is Borel summable if the path of integration from infinity to $x$ in (4.4) does not cross any Stokes curve.

See [DP, Theorem 1.2.2(c)] for the corresponding result for the Weber equation. Lemma 4.1 for the Whittaker equation can be verified in a similar manner.

Under our assumption we can choose a path of integration from infinity to $x$ in such a way that it does not cross any Stokes curve (see Remark below). Hence $\psi_{+, B}^{(\infty)}$ is holomorphic at $y=-y_{+}(x)+2 m \pi i \alpha$. This enables us to compute the alien derivative of $\psi_{+, B}$ at $y=-y_{+}(x)+2 m \pi i \alpha$ as follows:

$$
\begin{aligned}
\Delta_{y=-y_{+}(x)+2 m \pi i \alpha} \psi_{+, B} & =\Delta_{y=-y_{+}(x)+2 m \pi i \alpha}\left[\mathcal{B}\left(e^{V}\right) * \psi_{+, B}^{(\infty)}\right] \\
& =\left[\Delta_{y=-y_{+}(x)+2 m \pi i \alpha} \mathcal{B}\left(e^{V}\right)\right] * \psi_{+, B}^{(\infty)} \\
& =\left[\Delta_{y=-y_{+}(x)+2 m \pi i \alpha} V_{B}\right] \mathcal{B}\left(e^{V}\right) * \psi_{+, B}^{(\infty)} \\
& =2 \pi i \operatorname{Res}_{y=2 m \pi i \alpha}\left[V_{B}(\alpha, y)\right] \cdot \psi_{+, B} \\
& =\frac{e^{2 m \pi i \gamma}+e^{-2 m \pi i \gamma}}{2 m} \psi_{+, B} .
\end{aligned}
$$

This completes the proof of Theorem 4.1

Remark. If $x$ does not lie on a Stokes curve for $\arg \alpha=\pi / 2$, we can find a path of integration from infinity to $x$ so that it does not cross any Stokes curve for all $\alpha$ with $|\arg \alpha-\pi / 2|$ being sufficiently small. Examples of such paths are shown in Figures 8-10. For example, for $x_{j}(j=1,2)$ in Figure $8, \gamma_{j}$ gives a path satisfying the above condition.

\section{§4.2. Derivation of the Stokes automorphism for WKB solutions}

It follows from Theorem 4.1 that, when $\arg \alpha=\pi / 2$, the singular points $y=$ $-y_{+}(x)-2 m \pi i \alpha(m=1,2, \ldots)$ of the Borel transform of the WKB solution $\psi_{+, B}$ are located on

$$
\left\{y ; \Re y \geq \Re\left(-y_{+}(x)\right), \Im y=\Im\left(-y_{+}(x)\right)\right\} .
$$

As 4.5 is nothing other than the path of integration for the Borel sum of $\psi_{+}$, this implies that $\psi_{+}$is not Borel summable when $\arg \alpha=\pi / 2$. If we let the parameter $\alpha$ vary from $\arg \alpha=\pi / 2-\varepsilon$ to $\arg \alpha=\pi / 2+\varepsilon$, these singular points cross the path of integration for the Borel sum. Therefore, we conclude that the WKB 


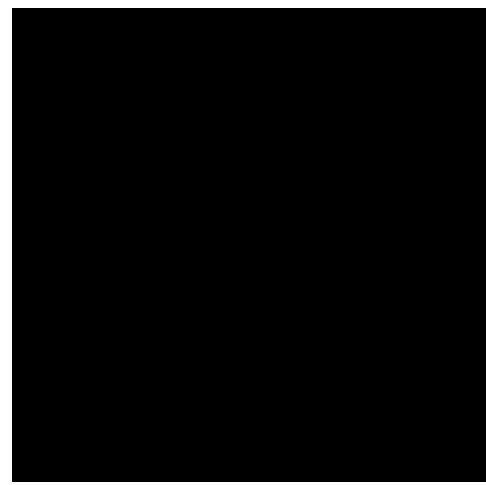

Figure 8. $\arg \alpha=\pi / 2$

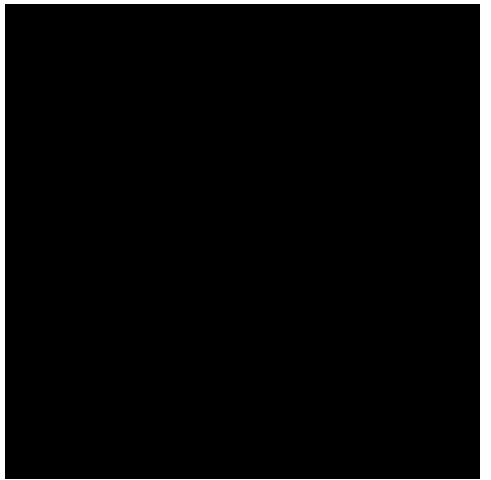

Figure $9 . \arg \alpha=\pi / 2-0.2$

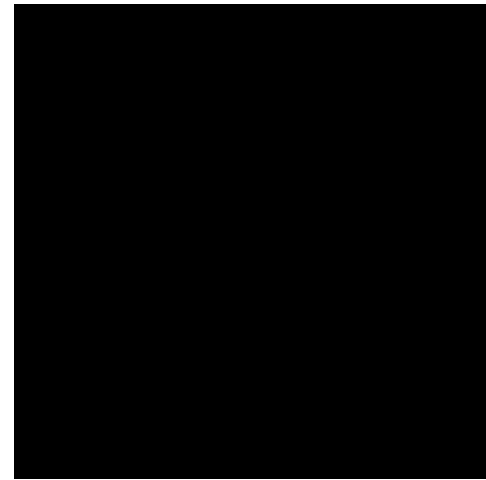

Figure 10. $\arg \alpha=\pi / 2+0.2$

solution $\psi_{+}$suffers a Stokes phenomenon (with respect to $\alpha$ ) at $\arg \alpha=\pi / 2$. Such Stokes phenomena are well described by the Stokes automorphism (cf. [Sa ). In this subsection we compute the action of the Stokes automorphism on the WKB solutions $\psi_{ \pm}$.

Let $\mathfrak{S}$ denote the Stokes automorphism. In our case, it is defined by

$$
\begin{aligned}
& \mathfrak{S} \psi_{+}=\exp \left[\sum_{m=1}^{\infty} \Delta_{y=-y_{+}(x)-2 m \pi i \alpha}\right] \psi_{+}, \\
& \mathfrak{S} \psi_{-}=\exp \left[\sum_{m=1}^{\infty} \Delta_{y=-y_{-}}(x)-2 m \pi i \alpha\right] \psi_{-} .
\end{aligned}
$$

Theorem 4.2. When $\arg \alpha=\pi / 2, \mathfrak{S} \psi_{ \pm}$are explicitly given by

$$
\mathfrak{S} \psi_{ \pm}=\left(1-e^{2 \pi i(\alpha \eta+\gamma)}\right)^{1 / 2}\left(1-e^{2 \pi i(\alpha \eta-\gamma)}\right)^{1 / 2} \psi_{ \pm} .
$$


Proof. We compute the action of the Stokes automorphism in the formal model. Let $\Delta$ be the sum of the alien derivatives:

We then obtain

$$
\Delta=\sum_{m=1}^{\infty} \Delta_{y=-y_{+}}(x)-2 m \pi i \alpha .
$$

$$
\begin{aligned}
\Delta \psi_{+} & =\sum_{m=1}^{\infty} \mathfrak{B}^{-1}\left[\Delta_{y=-y_{+}(x)-2 m \pi i \alpha} \psi_{+, B}\right] \\
& =-\sum_{m=1}^{\infty} \frac{e^{2 m \pi i \gamma}+e^{-2 m \pi i \gamma}}{2 m} \mathfrak{B}^{-1} \psi_{+, B}(x, y+2 m \pi i \alpha) \\
& =-\sum_{m=1}^{\infty} \frac{e^{2 m \pi i \gamma}+e^{-2 m \pi i \gamma}}{2 m} e^{2 m \pi i \alpha \eta} \psi_{+} \\
& =\frac{1}{2} \log \left[\left(1-e^{2 \pi i(\alpha \eta+\gamma)}\right)\left(1-e^{2 \pi i(\alpha \eta-\gamma)}\right)\right] \psi_{+} \cdot
\end{aligned}
$$

Here $\mathfrak{B}$ denotes the Borel transformation. Therefore we obtain

$$
\mathfrak{S} \psi_{+}=e^{\Delta} \psi_{+}=\left(1-e^{2 \pi i(\alpha \eta+\gamma)}\right)^{1 / 2}\left(1-e^{2 \pi i(\alpha \eta-\gamma)}\right)^{1 / 2} \psi_{+} \cdot
$$

The same computation can also be done for $\psi_{-}$.

\section{$\S 4.3$. Borel sum of the Voros coefficient}

In this final subsection we determine the Borel sum of the Voros coefficient.

Theorem 4.3. Let $\epsilon>0$ be sufficiently small. Then the Borel sum of the Voros coefficient $V_{B}$ for $\arg \alpha=\pi / 2-\varepsilon$ is given by

(4.6) $\frac{1}{2} \log \frac{\Gamma(\alpha \eta-\gamma)}{\sqrt{2 \pi}}+\frac{1}{2} \log \frac{\Gamma(\alpha \eta+\gamma)}{\sqrt{2 \pi}}+\frac{1}{2} \log (\alpha \eta+\gamma)+\alpha \eta(1-\log (\alpha \eta))$,

and for $\arg \alpha=\pi / 2+\varepsilon$ it is

$$
\begin{aligned}
-\frac{1}{2} \log \frac{\Gamma(-\alpha \eta-\gamma)}{\sqrt{2 \pi}}-\frac{1}{2} \log \frac{\Gamma(-\alpha \eta+\gamma)}{\sqrt{2 \pi}} \\
-\frac{1}{2} \log (-\alpha \eta+\gamma)+\alpha \eta(1-\log (\alpha \eta)+\pi i) .
\end{aligned}
$$

Proof. Making a change of variables $w=y / \alpha$, we obtain

$$
\begin{aligned}
\alpha V_{B}= & \frac{1}{2 w}\left[\frac{e^{w}}{e^{w}-1} e^{\gamma w}+\frac{1}{e^{w}-1} e^{-\gamma w}\right]-\frac{1}{w^{2}} \\
= & \frac{1}{2 w}\left(\frac{1}{e^{w}-1}+\frac{1}{2}-\frac{1}{w}\right) e^{\gamma w}+\frac{1}{2 w}\left(\frac{1}{e^{w}-1}+\frac{1}{2}-\frac{1}{w}\right) e^{-\gamma w} \\
& +\frac{\sinh (\gamma w)}{2 w}+\frac{\cosh (\gamma w)-1}{w^{2}}
\end{aligned}
$$


Let $\mathcal{S}[V]$ denote the Borel sum of the Voros coefficient $V$, i.e.,

$$
\mathcal{S}[V](\alpha, \eta):=\int_{0}^{\infty} e^{-y \eta} V_{B}(\alpha, y) d y=\int_{0}^{\infty} e^{-\alpha w \eta}\left(\alpha V_{B}(\alpha, \alpha w)\right) d w .
$$

To compute $\mathcal{S}[V](\alpha, \eta)$, we make use of the following formula:

$$
\int_{0}^{\infty}\left(\frac{1}{e^{t}-1}+\frac{1}{2}-\frac{1}{t}\right) e^{-t \theta} \frac{d t}{t}=\log \frac{\Gamma(\theta)}{\sqrt{2 \pi}}-\left(\theta-\frac{1}{2}\right) \log \theta+\theta \quad(\Re \theta>0)
$$

(cf. [E, Vol. I, Chapter I, $§ 1.9,(5)]$ ). We also use the following formulas for Laplace transforms of hyperbolic functions:

$$
\begin{aligned}
& \int_{0}^{\infty} e^{-p t} \frac{\sinh (a t)}{t} d t=\frac{1}{2} \log \frac{p+a}{p-a}, \\
& \int_{0}^{\infty} e^{-p t} \frac{\cosh (a t)-1}{t^{2}} d t=\frac{a}{2} \log \frac{p+a}{p-a}+\frac{p}{2} \log \left(1-\frac{a^{2}}{p^{2}}\right) .
\end{aligned}
$$

Then, when $\arg \alpha=\pi / 2-\varepsilon$, we find

$$
\begin{aligned}
\mathcal{S}[V](\alpha, \eta)= & \frac{1}{2}\left[\frac{\Gamma(\eta \alpha-\gamma)}{\sqrt{2 \pi}}-\left(\eta \alpha-\gamma-\frac{1}{2}\right) \log (\eta \alpha-\gamma)+\eta \alpha-\gamma\right] \\
& +\frac{1}{2}\left[\frac{\Gamma(\eta \alpha+\gamma)}{\sqrt{2 \pi}}-\left(\eta \alpha+\gamma-\frac{1}{2}\right) \log (\eta \alpha+\gamma)+\eta \alpha+\gamma\right] \\
& +\frac{1}{4} \log \frac{\eta \alpha+\gamma}{\eta \alpha-\gamma}+\frac{\gamma}{2} \log \frac{\eta \alpha+\gamma}{\eta \alpha-\gamma}+\frac{\eta \alpha}{2} \log \left(1-\frac{\gamma^{2}}{\eta^{2} \alpha^{2}}\right) \\
= & \frac{1}{2} \log \frac{\Gamma(\alpha \eta-\gamma)}{\sqrt{2 \pi}}+\frac{1}{2} \log \frac{\Gamma(\alpha \eta+\gamma)}{\sqrt{2 \pi}} \\
& +\frac{1}{2} \log (\alpha \eta+\gamma)+\alpha \eta(1-\log (\alpha \eta)) .
\end{aligned}
$$

When $\arg \alpha=\pi / 2-\varepsilon$, we can compute $\mathcal{S}[V](\alpha, \eta)$ in a similar manner by using the relation $\mathcal{S}[V](\alpha, \eta)=-\mathcal{S}[V](-\alpha, \eta)$.

Using the concrete expressions 4.6 and 4.7) for the Borel sum of $V$ given by Theorem 4.3 , we can prove Theorem 4.2 without resorting to the alien calculus. We conclude this final section with a different proof of Theorem 4.2 which is based on Theorem 4.3 .

Since both $\psi_{+}^{(\infty)}$ and $V$ are Borel summable for $\arg \alpha=\pi / 2 \pm \varepsilon$, it follows from 4.3 that

$$
\begin{aligned}
& \left.\mathcal{S}\left[\psi_{+}\right]\right|_{\arg \alpha=\pi / 2-\varepsilon}=\left.\exp \left[\left.\mathcal{S}[V]\right|_{\arg \alpha=\pi / 2-\varepsilon}\right] \cdot \mathcal{S}\left[\psi_{+}^{(\infty)}\right]\right|_{\arg \alpha=\pi / 2-\varepsilon}, \\
& \left.\mathcal{S}\left[\psi_{+}\right]\right|_{\arg \alpha=\pi / 2+\varepsilon}=\left.\exp \left[\left.\mathcal{S}[V]\right|_{\arg \alpha=\pi / 2+\varepsilon}\right] \cdot \mathcal{S}\left[\psi_{+}^{(\infty)}\right]\right|_{\arg \alpha=\pi / 2+\varepsilon},
\end{aligned}
$$


where $\mathcal{S}$ denotes the Borel sum. Note that $\psi_{+}^{(\infty)}$ is Borel summable for $\arg \alpha \in$ $[\pi / 2-\varepsilon, \pi / 2+\varepsilon]$ for a sufficiently small positive $\varepsilon$ in view of Lemma 4.1. Thus we have

$$
\left.\mathcal{S}\left[\psi_{+}^{(\infty)}\right]\right|_{\arg \alpha=\pi / 2+\varepsilon}=\left.\mathcal{S}\left[\psi_{+}^{(\infty)}\right]\right|_{\arg \alpha=\pi / 2-\varepsilon}
$$

Therefore, we obtain

$$
\frac{\left.\mathcal{S}\left[\psi_{+}\right]\right|_{\arg \alpha=\pi / 2+\varepsilon}}{\left.\mathcal{S}\left[\psi_{+}\right]\right|_{\arg \alpha=\pi / 2-\varepsilon}}=\exp \left[\left.\mathcal{S}[V]\right|_{\arg \alpha=\pi / 2+\varepsilon}-\left.\mathcal{S}[V]\right|_{\arg \alpha=\pi / 2-\varepsilon}\right] .
$$

The right-hand side of 4.8 can be explicitly computed thanks to Theorem 4.3 .

$$
\begin{aligned}
\left.\mathcal{S}[V]\right|_{\arg \alpha=\pi / 2+\varepsilon} & -\left.\mathcal{S}[V]\right|_{\arg \alpha=\pi / 2-\varepsilon} \\
= & -\frac{1}{2} \log \frac{\Gamma(-\alpha \eta-\gamma) \Gamma(-\alpha \eta+\gamma) \Gamma(\alpha \eta-\gamma) \Gamma(\alpha \eta+\gamma)}{(2 \pi)^{2}} \\
& -\frac{1}{2} \log \left(\gamma^{2}-\alpha^{2} \eta^{2}\right)+\pi i \alpha \eta
\end{aligned}
$$

Since

$$
\frac{\Gamma(\alpha \eta-\gamma) \Gamma(-\alpha \eta+\gamma)}{2 \pi}=\frac{1}{-\alpha \eta+\gamma} \frac{1}{2 \sin \pi(\alpha \eta-\gamma)}
$$

and

$$
\frac{\Gamma(\alpha \eta+\gamma) \Gamma(-\alpha \eta-\gamma)}{2 \pi}=\frac{1}{-\alpha \eta-\gamma} \frac{1}{2 \sin \pi(\alpha \eta+\gamma)}
$$

the right-hand side of 4.9 becomes

$$
\begin{aligned}
\frac{1}{2} \log \frac{4\left(\alpha^{2} \eta^{2}-\gamma^{2}\right) \sin \pi(\alpha \eta+\gamma) \sin \pi(\alpha \eta-\gamma)}{\gamma^{2}-\alpha^{2} \eta^{2}}+\pi i \alpha \eta \\
\quad=\frac{1}{2} \log \left[\left(e^{i \pi(\alpha \eta+\gamma)}-e^{-i \pi(\alpha \eta+\gamma)}\right)\left(e^{i \pi(\alpha \eta-\gamma)}-e^{-i \pi(\alpha \eta-\gamma)}\right)\right]+\pi i \alpha \eta \\
=\frac{1}{2} \log \left[\left(1-e^{2 i \pi(\alpha \eta+\gamma)}\right)\left(1-e^{2 i \pi(\alpha \eta-\gamma)}\right)\right]
\end{aligned}
$$

Hence we obtain

$$
\left.\mathcal{S}\left[\psi_{+}\right]\right|_{\arg \alpha=\pi / 2+\varepsilon}=\left.\left(1-e^{2 i \pi(\alpha \eta+\gamma)}\right)^{1 / 2}\left(1-e^{2 i \pi(\alpha \eta-\gamma)}\right)^{1 / 2} \mathcal{S}\left[\psi_{+}\right]\right|_{\arg \alpha=\pi / 2-\varepsilon} .
$$

This proves Theorem 4.2 .

\section{Acknowledgments}

We would like to express our sincere gratitude to Professor Takahiro Kawai and Professor Takashi Aoki for their kind encouragement and stimulating discussions.

The research of the authors has been supported in part by JSPS grants-in-aid No. 21740098, No. 21340029 and No. S-19104002. 


\section{References}

[AKT1] T. Aoki, T. Kawai and Y. Takei, The Bender-Wu analysis and the Voros theory, in Special functions, Springer, 1991, 1-29. Zbl 0782.35060 MR 1166808

[AKT2] , The Bender-Wu analysis and the Voros theory II, Adv. Stud. Pure Math. 54 (2009), 19-94. Zbl 1175.34112 MR 2499553

[DDP] E. Delabaere, H. Dillinger and F. Pham, Résurgence de Voros et périodes des courbes hyperelliptiques, Ann. Inst. Fourier (Grenoble) 43 (1993), 163-199. Zbl 0766.34032 MR 1209700

[DP] E. Delabaere and F. Pham, Resurgent methods in semi-classical asymptotics, Ann. Inst. H. Poincaré Phys. Théor. 71 (1999), 1-94. Zbl 0977.34053 MR 1704654

[E] A. Erdélyi et al., Higher transcendental functions, Bateman Manuscript Project (Vol. I, II, III), Krieger, Malabar, FL, 1981. Zbl 0542.33001(Vol. I), Zbl 0505.33001(Vol. II), Zbl 0542.33002(Vol. III) MR 0698779(Vol. I), MR 0698780(Vol. II), MR 0698781(Vol. III)

[KKKoT1] S. Kamimoto, T. Kawai, T. Koike and Y. Takei, On the WKB theoretic structure of a Schrödinger operator with a merging pair of a simple pole and a simple turning point, Kyoto J. Math. 50 (2010), 101-164. MR 2629645

[KKKoT2] _ _ On a Schrödinger equation with a merging pair of a simple pole and a simple turning point-Alien calculus of WKB solutions through microlocal analysis, preprint RIMS-1686, 2009.

[KT1] T. Kawai and Y. Takei, Secular equations through the exact WKB analysis, in Analyse algébrique des perturbations singulières, I, Méthodes résurgentes, Hermann, 1994, 85-102. Zbl 0834.34068 MR 1296473

[KT2] Algebraic analysis of singular perturbation theory, Transl. Math. Monogr. 227, Amer. Math. Soc., Providence, RI, 2005. MR 2182990

[Ko1] T. Koike, On a regular singular point in the exact WKB analysis, in Toward the exact WKB analysis of differential equations, linear or non-linear, Kyoto Univ. Press, 2000, 39-54. Zbl 0986.34076 MR 1770282

[Ko2] _ On the exact WKB analysis of second order linear ordinary differential equations with simple poles, Publ. RIMS Kyoto Univ. 36 (2000), 297-319. Zbl 0972.34078 MR 1753205

[Sa] D. Sauzin, Resurgent functions and splitting problems, RIMS Kôkyûroku 1493 (2006), 48-117.

[SS] H. Shen and H. J. Silverstone, Observations on the JWKB treatment of the quadratic barrier, in Algebraic analysis of differential equations, Springer, 2007, 237-250. Zbl 1145.34384

[T] Y. Takei, Sato's conjecture for the Weber equation and transformation theory for Schrödinger equations with a merging pair of turning points, RIMS Kôkyûroku Bessatsu B10 (2008), 205-224. Zbl 1170.34064 MR 2498081

[V] A. Voros, The return of the quartic oscillator-The complex WKB method, Ann. Inst. H. Poincaré Sect. A 39 (1983), 211-338. Zbl 0526.34046 MR 0729194 